\title{
Abrogation of p53 function leads to metastatic transcriptome networks that typify tumor progression in human breast cancer xenografts
}

\author{
ANTONINO B. D'ASSORO ${ }^{1,6}$, ALEXEY LEONTOVICH ${ }^{2}$, ANGELA AMATO ${ }^{1}$, JENNIFER R. AYERS-RINGLER ${ }^{1}$, \\ COSIMA QUATRARO ${ }^{1}$, KARI HAFNER ${ }^{3}$, ROBERT B. JENKINS ${ }^{3}$, MASSIMO LIBRA ${ }^{6}$, JAMES INGLE ${ }^{4}$, \\ FRANCA STIVALA $^{6}$, EVANTHIA GALANIS ${ }^{4,5}$ and JEFFREY L. SALISBURY ${ }^{1}$ \\ ${ }^{1}$ Department of Biochemistry and Molecular Biology, ${ }^{2}$ Division of Biomedical Statistics and Informatics, ${ }^{3}$ Laboratory \\ Medicine and Pathology, Departments of ${ }^{4}$ Medical Oncology, and ${ }^{5}$ Molecular Medicine, Mayo Clinic School of Medicine, \\ Rochester, MN 55905, USA; ${ }^{6}$ Department of Biomedical Sciences, University of Catania, Catania 95123, Italy
}

Received July 9, 2010; Accepted August 2, 2010

DOI: 10.3892/ijo_00000768

\begin{abstract}
Development of chromosomal instability (CIN) and consequent phenotypic heterogeneity represent common events during breast cancer progression. Breast carcinomas harboring extensive chromosomal aberrations display a more aggressive behavior characterized by chemoresistance and the propensity to give rise to distant metastases. The tumor suppressor p53 plays a key role in the maintenance of chromosomal stability and tissue homeostasis through activation of cell cycle checkpoints following DNA damage and control of centrosome duplication that ensures equal chromosome segregation during cell division. Furthermore, p53 suppresses CD44 expression and the acquisition of stem cell-like properties responsible for epithelial to mesenchymal transition (EMT) and metastasis. In this study we employed MCF-7 breast cancer cells with endogenous wild-type p53, an engineered MCF-7 variant (vMCF-7 ${ }^{\text {DNP53}}$ ) overexpressing a dominant negative p53val135 mutant, and cells re-cultured from vMCF-7 ${ }^{\text {DNP53 }}$ tumor xenografts. We carried out an integrative transcriptome and cytogenetic analysis to characterize the mechanistic linkage between loss of p53 function,
\end{abstract}

Correspondence to: Dr Jeffrey L. Salisbury, Tumor Biology Program, Mayo Clinic College Of Medicine, 200 First Street SW, Rochester, MN 55905, USA

E-mail: salisbury@mayo.edu

Abbreviations: CIN, chromosomal instability; EGF, epidermal growth factor; EMT, epithelial mysenchymal transition; ER, estrogen receptor; IGFBP5, insulin-like growth factor binding protein-5; SKY, spectral karyotyping analysis; TGF, transforming growth factor.

Key words: breast cancer, centrosome amplification, chromosomal instability, epithelial mysenchymal transition, invasive gene networks, tumor cell heterogeneity, tumor progression
EMT and consequent establishment of invasive gene signatures during breast cancer progression. We demonstrate that abrogation of p53 function drives the early transcriptome changes responsible for cell proliferation, EMT and survival, while further transcriptome changes that occur during in vivo tumor progression are mechanistically linked to the development of CIN leading to a more invasive and metastatic breast cancer phenotype. Here we identified distinct novel non-canonical transcriptome networks involved in cell proliferation, EMT, chemoresistance and invasion that arise following abrogation of p53 function in vitro and development of CIN in vivo. These studies also have important translational implications since some of the nodal genes identified here are 'druggable' making them appropriate molecular targets for the treatment of breast carcinomas displaying mutant p53, EMT, CIN and high metastatic potential.

\section{Introduction}

Human breast tumors displaying an aggressive behavior are generally characterized by high tumor cell heterogeneity, which has been associated with the development of chromosomal instability (CIN) during tumor progression $(1,2)$. Chromosomal instability represents a hallmark of solid tumors and is characterized by the high frequency of qualitative and quantitative chromosome abnormalities that arise during tumor progression (3). Centrosome duplication plays a critical role in the control of chromosomal stability through the establishment of bipolar mitotic spindles and propagation of a diploid karyotype $(4,5)$. In normal cells, centrosome duplication is coordinated with DNA replication, ensuring the establishment of bipolar mitotic spindle leading to equal chromosome segregation (6-8). Although chromosomal instability may be present during the early stages of neoplastic transformation, the persistence of CIN during tumor progression and its causative role in the development of distant metastases, chemoresistance, and poor outcome are currently highly debated. This notwithstanding, breast tumors are thought to become refractory to conventional anti-cancer 
therapies due in part to tumor cell heterogeneity, which confers manifold options for resistance to a given treatment modality (9-11). The tumor suppressor p53, whose function is lost in $50 \%$ of human cancers, plays a key role in the maintenance of chromosomal stability through tight coordination of DNA replication with centrosome duplication, activation of cell cycle checkpoints following genotoxic stress, and initiation of programmed cell death if damaged DNA can not be repaired properly $(5,7,12,13)$. P53 operates as a tumor suppressor mainly as a transcription factor that directly binds to the promoter region of target genes and activates or suppresses their transcription (14). Importantly, centrosome homeostasis is disrupted in cancer cells lacking p53 function due to deregulated activity of cdk 2 kinase and consequent abrogation of cell cycle checkpoints resulting in centrosome amplification and CIN $(7,15,16)$. In breast cancer, absence of p53 function has been associated with an aggressive phenotype characterized by CIN, hormone independence, resistance to anticancer drugs and poor prognosis (17). Breast tumors with basal-like phenotype and those overexpressing the HER-2/ Neu receptor usually display a mutant p53 $(18,19)$. The hypothesis that loss of p53 function favors tumor progression and metastasis through the development of chromosomal instability and consequent tumor cell heterogeneity is supported by several studies. In a p53-null mouse model for mammary tumorigenesis, impaired p53 function accelerates the development of CIN and tumor cell heterogeneity characterized by the loss of ER expression (20). Similarly, impaired p53 function leads to centrosome amplification, aberrant mitoses, development of high-grade breast tumors, ER tumor cell heterogeneity and distant metastases in an ER positive human breast cancer xenograft model (12). Recent studies have also implicated a novel role for p53 in the inhibition of tumor progression through suppression of CD44 expression and epithelial to mesenchymal transition (EMT) responsible for tumor self-renewal and metastasis (21). Taken together, these findings demonstrate that p53 interferes with the development of tumor cell heterogeneity responsible for the invasive phenotype by maintaining chromosomal stability and by suppressing EMT. However, the molecular mechanisms linking loss of p53 function to development of chromosomal instability, phenotypic heterogeneity, and distant metastases remain largely unknown.

In this study we employed MCF-7 cells with endogenous wild-type p53, variant MCF-7 cells (vMCF-7 ${ }^{\text {DNP53 }}$ ) engineered to overexpress a dominant negative p53val135 mutant, and cells re-cultured from vMCF-7 ${ }^{\text {DNP53 }}$ tumor xenografts. We carried out a whole genome microarray analysis and an integrative cytogenetic approach to characterize the mechanistic linkage between loss of p53 function, development of EMT and metastatic transcriptome signatures that characterize tumor progression.

\section{Materials and methods}

Human breast cancer cell lines. The human breast cancer cell line MCF-7 (ATCC, Manassas, VA, USA), was modified to express a recombinant temperature sensitive p53 construct mutated at residue 135 to valine (vMCF- ${ }^{\mathrm{DNp} 53}$ ) as described previously $(7,12,22)$. Cultures were maintained in EMEM medium containing $5 \mathrm{mM}$ glutamine, $1 \%$ penicillin/ streptomycin, $20 \mu \mathrm{g} / \mathrm{ml}$ insulin and $10 \% \mathrm{FBS}$ at $37^{\circ} \mathrm{C}$ in $5 \% \mathrm{CO}_{2}$, and the engineered clones were maintained under $500 \mu \mathrm{g} / \mathrm{ml}$ G418 selection. In some experiments cultures were treated with $1 \mu \mathrm{M}$ doxorubicin in DMSO $48 \mathrm{~h}$ to induce genotoxic stress and apoptosis, or DMSO alone (1:1000).

Fluorescence microscopy. Cells were fixed in absolute methanol at $-20^{\circ} \mathrm{C}$ for $10 \mathrm{~min}$, blocked in $5 \%$ normal goat serum, $1 \%$ glycerol, $0.1 \%$ BSA, $0.1 \%$ fish skin gelatin, $0.04 \%$ sodium azide and incubated with primary antibodies. Primary antibodies against the proteins centrin (20H5 or hCetn-2.4 produced in our laboratory), $\gamma$-tubulin (Sigma), pericentrin (Sigma), and Aurora-A (Santa Cruz) were followed by secondary antibodies conjugated with Alexa 488 or Alexa 568 (Molecular Probes, Eugene, OR, USA). GFPlabeled centrioles were counted in cells fixed in $4 \%$ formaldehyde, incubated in Hoescht dye at $1 \mu \mathrm{g} / \mathrm{ml}$ to stain DNA, and mounted using ProLong antifade (Molecular Probes). Images were digitally recorded at multiple focal planes using a Zeiss Axiovert $200 \mathrm{M}$ fluorescence microscope and analyzed as maximum projections. Values reported represent the average of 100 cells in each of two independent experiments.

Immunoblotting. Cell lysates (20 $\mu \mathrm{g}$ protein) were run in $12 \%$ SDS-PAGE, transferred to PVDF membrane, fixed in $0.25 \%$ glutaraldehyde, blocked in 5\% non-fat dry milk and incubated with primary antibodies against the following proteins: p53 (D07 Dako), p21 (oncogene), Mdm-2 (Santa-Cruz), phosphoretinoblastoma (Sigma, St. Louis, MO, USA), cyclins D1, E, A (Santa Cruz) and $\beta$-actin (Sigma) as loading control, followed by HRP secondary antibodies (Amersham, Piscataway, NJ, USA), and detected using the ECL-plus reagent (Amersham) and a UVP BioImaging system.

Xenograft models. Procedures based on US NIH guidelines for the care and use of laboratory animals were followed for all experiments and were approved by the Mayo Institutional Animal Care and Use Committee (IACUC Protocol A34008). Four-week-old non-ovariectomized female NCR/Nu/Nu nude mice were anesthetized by exposure to $3 \%$ isoflurane and injected subcutaneously (s.c) with $2 \times 10^{6}$ cells suspended in $50 \mu \mathrm{l}$ of $50 \%$ Matrigel (BD Bioscience, Bedford). After 12 weeks mice were sacrificed and xenograft tumors were processed for molecular and cell biological analyses. To reculture first generation xenografts (1GX) explants, tumors were excised from sacrificed animals, minced using sterile scissors, transferred to complete culture medium, and fibroblast-free tumor cell lines were re-established by serial passages in culture.

Gene microarray analysis. Total RNA was extracted from breast cancer cells using TRizol according to the manufacturer's instructions (Invitrogen, Carlsbad, CA). One microgram of total RNA (A260/A280 ratio of 1.8-2.2) was used to probe for global genome expression employing Affimetrix U133 Plus 2.0 chips (Affimetrix, Santa Clara, CA). Gene network analysis was performed using Ingenuity Pathways Analysis software (Redwood City, CA). Experiments were performed in duplicate. 

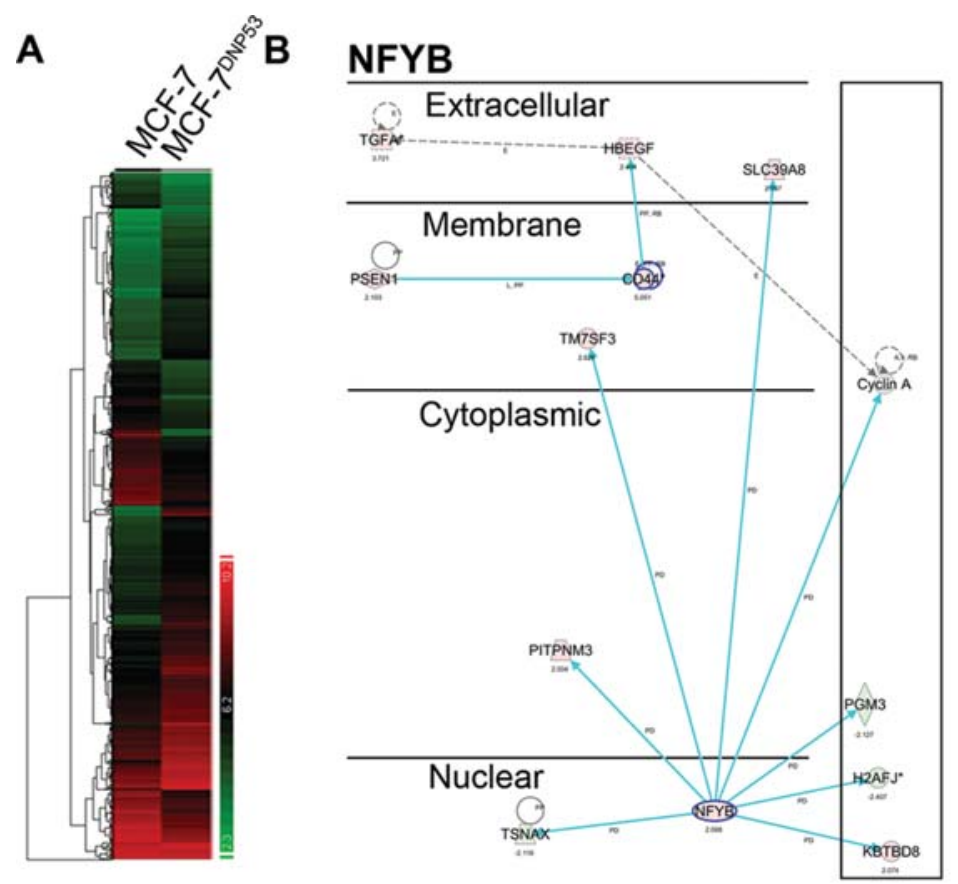

\section{SMAD3}

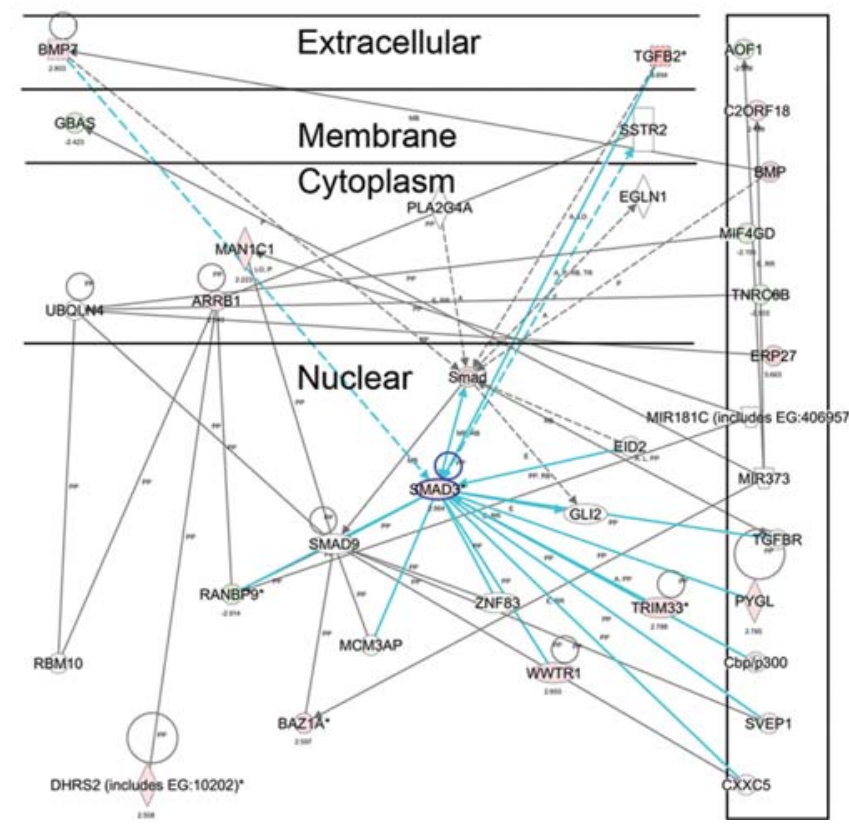

Figure 1. Global gene expression profile and non-canonical transcriptome networks in human breast cancer cell lines. (A) Heat map representing the unsupervised cluster analysis of global gene expression in MCF-7 and vMCF-7 ${ }^{\mathrm{DNp} 53}$ cells. The genes were selected based on 2-fold change cut-off. Data set used for hierarchical clustering were normalized by standardizing the expression level of each gene and each sample to mean $=0$ and variance $=1$. The diagram shows consistent changes between two separate analyses. (B and C) Non-canonical gene network maps identified in the comparison of gene expression between MCF-7 and vMCF-7 ${ }^{\mathrm{DNp} 53}$ cells. Gene network analysis using Ingenuity Pathways Analysis software program identified major noncanonical gene networks where key nodal genes are involved in survival (NFYB), and EMT (SMAD3).

Cytogenetic and sky analysis. Cell harvest and metaphase slide preparation for routine cytogenetic and spectral karyotyping (SKY) analysis were performed as previously described (23-25). Hybridization, wash, and detection of the human SKYPaint ${ }^{\circledR}$ probe (Applied Spectral Imaging; Vista, CA) were performed as recommended by the manufacturer. Image acquisition and spectral analysis of metaphase cells were achieved by using the SD200 SpectraCube ${ }^{\text {TM }}$ Spectral Imaging system (Applied Spectral Imaging) mounted on a Zeiss
Axioplan2 microscope (Carl Zeiss MicroImaging, Inc., Thornwood, NY). Images were analyzed using HiSKY analysis software (Applied Spectral Imaging).

\section{Results and Discussion}

Microarray analysis of global genome expression identified 1655 genes that were differentially expressed between vMCF-7 ${ }^{\mathrm{DNP} 53}$ and parental MCF-7 cells (Fig. 1A). To determine 
A

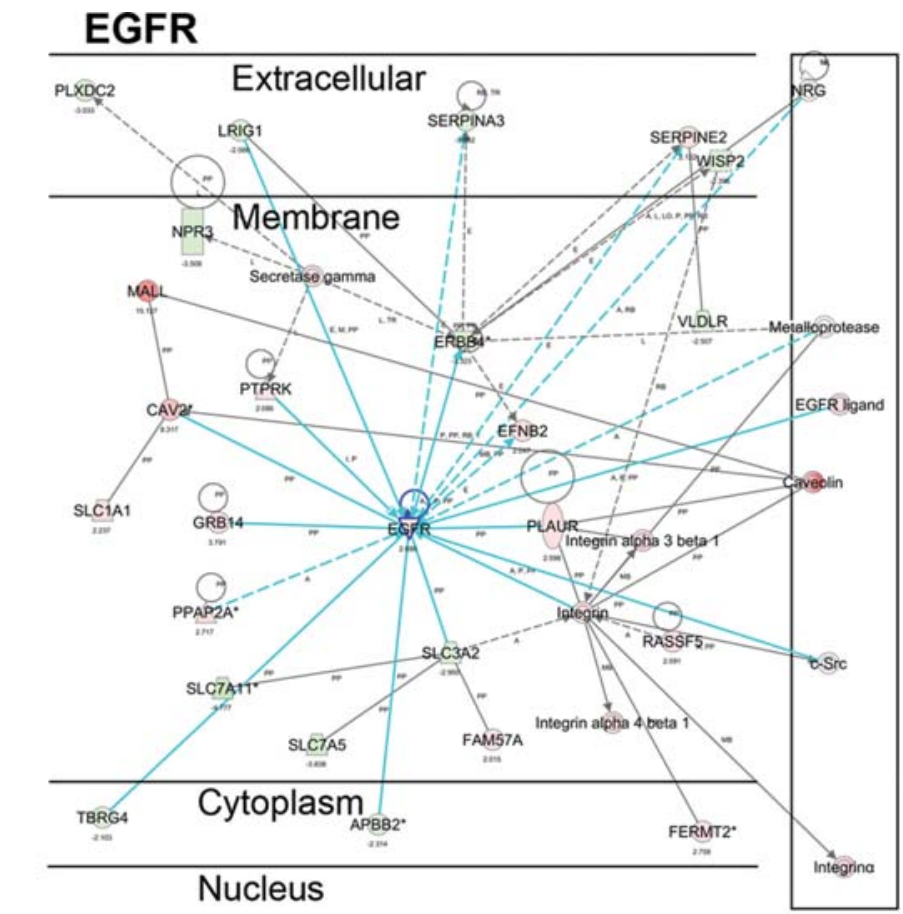

B

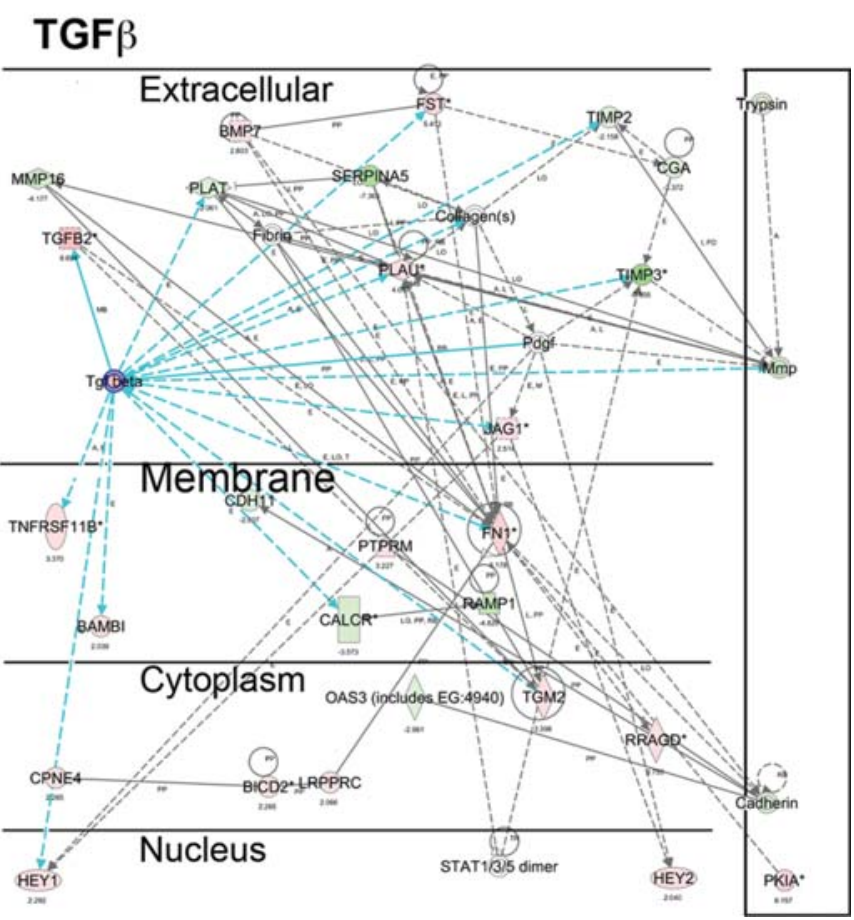

Figure 2. Non-canonical transcriptome networks in human breast cancer cell lines. (A and B) Non-canonical gene network maps identified in the comparison of gene expression between MCF-7 and vMCF-7 DNp53 $^{\mathrm{N}}$ cells. Gene network analysis using Ingenuity Pathways Analysis software program identified major non-canonical gene networks where key nodal genes are involved in cell proliferation (EGFR), and EMT (TGFß).

the presence of functional interactions between genes involved in cancer development and progression among the pool of 1655 genes identified, we performed a gene network analysis, which revealed four major non-canonical functional networks consisting of cancer related genes implicated in breast cancer progression where key nodal genes involved in cell proliferation (EGFR), survival (NFYB), and EMT (TGFß and SMAD3) were up-regulated in vMCF-7 ${ }^{\text {DNP53 }}$ compared to
MCF-7 parental cells (Fig. 1B and C and Fig. 2A and B). In particular, vMCF-7 DNP53 $^{\text {cells developed a transcriptome }}$ signature characterized by increased expression of oncogenes such as EGF receptor, NFYB, CD44, TGFB and SMAD3 and repression of tumor suppressors such as E-Cadherin, TIMP2 and TIMP3. These results indicate that introduction of mutant p53 into MCF-7 cells in vitro results in a transcriptome signature associated with loss of a luminal phenotype, 
A

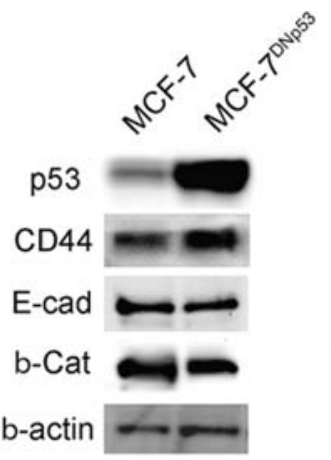

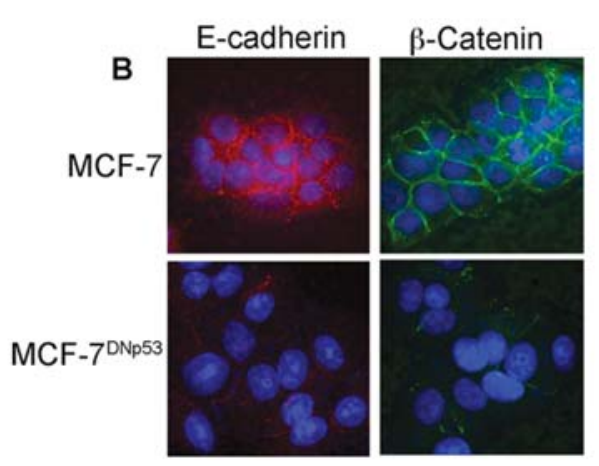
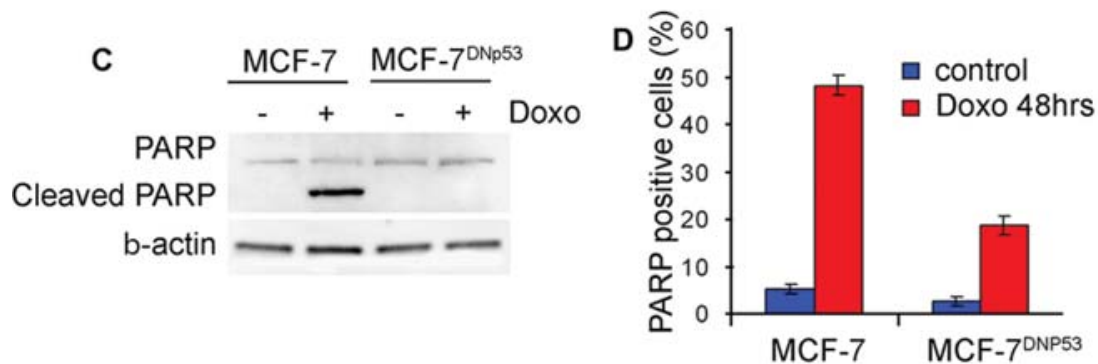

E
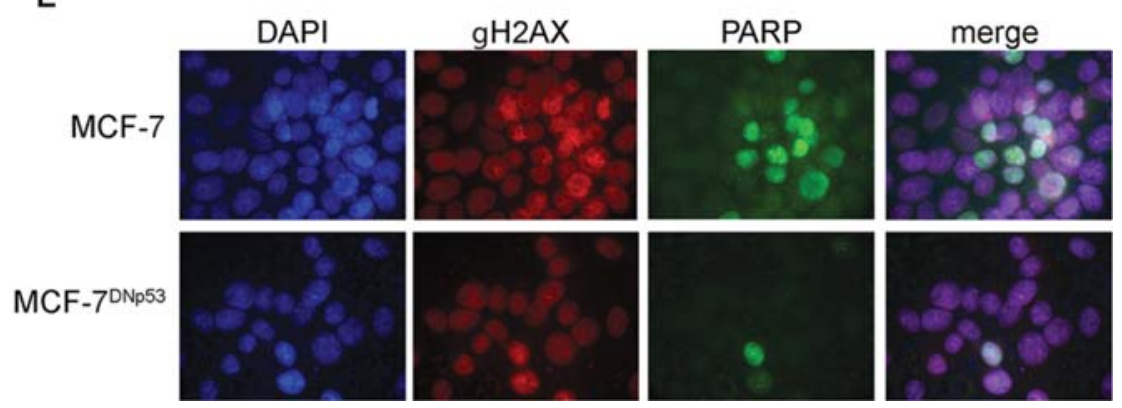

Figure 3. Characterization of EMT and chemoresistance in human breast cancer cell lines. (A) Western blot analysis showing that vMCF-7DNp53 cells overexpress the cancer stem cell marker CD44 (Abcam 24504, Cambridge MA) and reduced expression of epithelial markers E-cadherin and B-catenin (Santa Cruz SC8426 and SC7963, Santa Cruz, CA) compared to parental MCF-7 cells. (B) Immunofluorescence analysis shows loss of epithelial markers E-cadherin (red) and ß-catenin (green) in vMCF-7 ${ }^{\text {DNp53 }}$ cells compared to MCF-7 cells. Nuclei were stained in blue with Hoechst dye. (C) Western blot analysis of MCF-7 and vMCF-7 $7^{\mathrm{DNp} 53}$ cells treated $\pm 1 \mu \mathrm{M}$ doxorubicin for $48 \mathrm{~h}$. Induction of apoptosis after doxorubicin treatment was detected through PARP cleavage only in MCF-7 cells. (D) Graph showing the percentage of PARP positive cells detected by immunofluorescence following doxorubicin treatment from three independent experiments \pm SD. (E) Immunofluorescence analysis showing that while doxorubicin treatment induced DNA damage in both vMCF-7 $7^{\text {DNP53 }}$ and parental cells as indicated by $\gamma \mathrm{H} 2 \mathrm{AX}$ nuclear localization (red), only MCF-7 cells displayed a higher percentage of cells showing nuclear cleaved PARP (green). Nuclei were stained in blue with Hoechst dye.

increased cell proliferation and survival, and gain of a more invasive behavior. To validate cancer transcriptome changes identified by gene microarray analysis we performed immunoblot analysis that confirmed overexpression of the EMT marker CD44 surface receptor and reduced expression of epithelial markers E-cadherin and B-catenin in vMCF-7DNP53 cells compared to parental MCF-7 cells (Fig. 3A). Immunofluorescence analysis also showed loss of E-cadherin and Bcatenin cell membrane localization in vMCF-7DNP53 cells compared to parental cells (Fig. 3B). The majority of these transcriptome and phenotypic changes arise independent of chromosomal instability, since we have previously demonstrated that cultured vMCF-7 DNP53 $^{\text {show a normal centrosome }}$ and mitotic spindle phenotypes, and that centrosome amplification and aberrant mitoses develop in vitro only following genotoxic stress or in the context of in vivo tumor growth (12).

Since loss of p53 function and development of EMT in breast cancer is also associated with increased cell survival and chemoresistance, we determined the genotoxic sensitivity of vMCF-7 $7^{\mathrm{DNP53}}$ cells treated with daunorubicin compared to the parental MCF-7 and determined the presence of cleaved PARP as a marker of activation of programmed cell death. Treatment with doxorubicin induced PARP cleavage only in MCF-7 cells detectable by Western blot analysis (Fig. 3C). Likewise, at the cellular level doxorubicin treatment induced DNA damage in both vMCF-7 ${ }^{\text {DNP53 }}$ and parental cells as indicated by $\gamma \mathrm{H} 2 \mathrm{AX}$ nuclear localization, while only MCF-7 cells displayed a higher percentage of cells showing nuclear cleaved PARP (Fig. 3D and E). Taken together, these results demonstrate that mutant p53 leads to the development of an EMT phenotype and chemoresistance in cultured cells.

In order to characterize additional transcriptome changes that may arise in breast cancer cells lacking p53 function during tumor progression in vivo we compared the global gene expression profile between MCF-7, vMCF- $7^{\text {DNP53 }}$, and tumor cells re-cultured as first generation from vMCF-7DNP53 
A

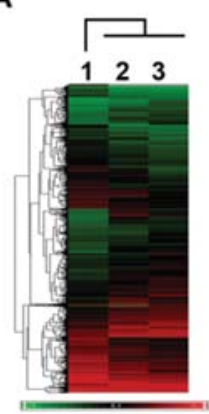

B

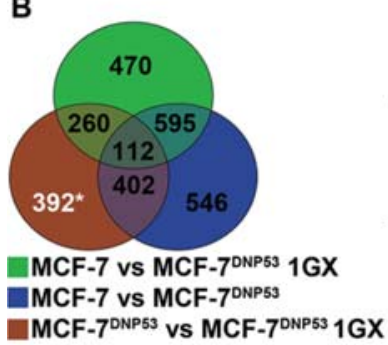

C

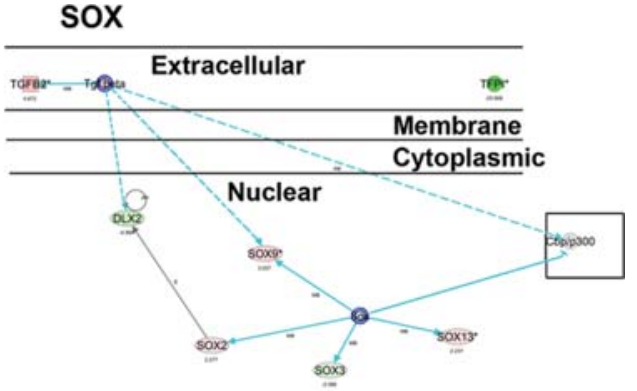

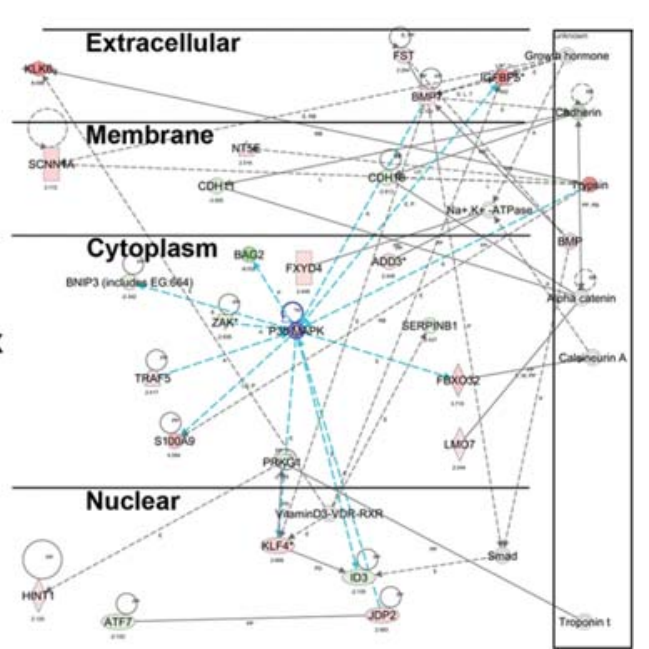

Figure 4. Global gene expression profile and non-canonical transcriptome networks in human breast cancer cell lines. (A) Heat map representing the

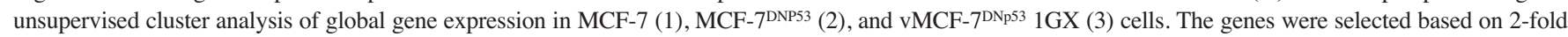
change cut-off. Data set used for hierarchical clustering were normalized by standardizing the expression level of each gene and each sample to mean $=0$ and variance $=1$. The diagram shows consistent changes between two separate analyses. (B) Venn diagram showing gene expression comparison between MCF-7, vMCF-7 $7^{\mathrm{DNp} 53}$ and vMCF-7 ${ }^{\mathrm{DNp} 53} 1 \mathrm{GX}$ cells. (C and D) Non-canonical transcriptome network maps identified in the comparison between vMCF-7 $7^{\mathrm{DNp} 53}$ and vMCF-7 ${ }^{\mathrm{DNp} 53} 1 \mathrm{GX}$ cells. Ingenuity Pathways Analysis program identifed major functional gene networks with important implications for breast cancer progression showing key nodal genes involved in tumor self-renewal (SOX), chemoresistance and metastases (p38MAPK signaling pathway) were overexpressed in vMCF-7 $7^{\mathrm{DNp} 53} 1 \mathrm{GX}$ cells compared to the vMCF-7 ${ }^{\mathrm{DNp} 53}$ parental cells.

\section{A IGFBP5}

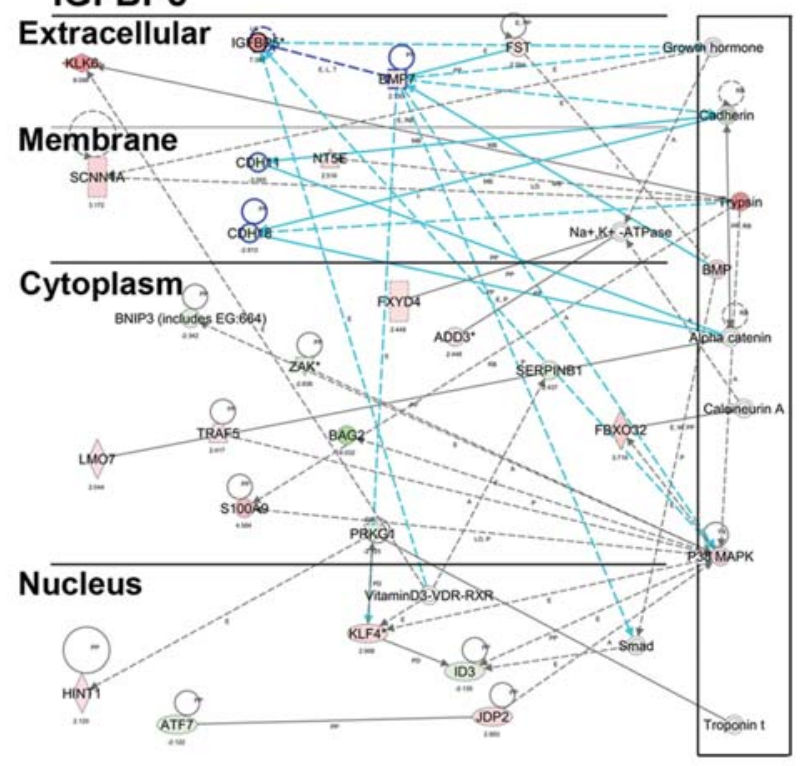

B Proteasome

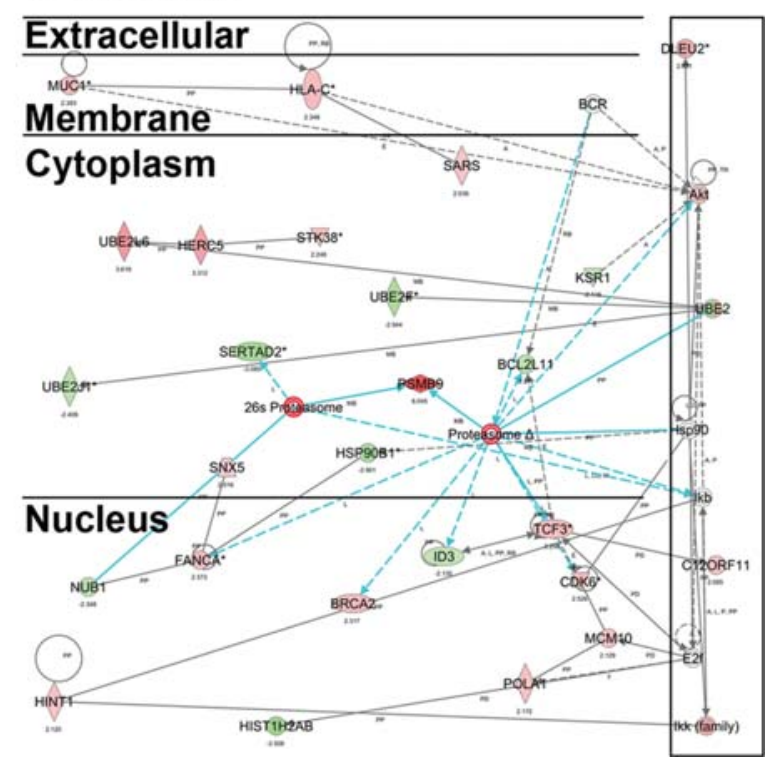

Figure 5. Non-canonical transcriptome networks in human breast cancer cell lines. (A and B) Non-canonical transcriptome network maps identified in the comparison between vMCF-7 ${ }^{\mathrm{DNp} 53}$ and vMCF-7 ${ }^{\mathrm{DNp} 53} 1 \mathrm{GX}$ cells. Ingenuity Pathways Analysis program identifed major functional gene networks with important implications for breast cancer progression showing key nodal genes involved in chemoresistance and metastases (IGFB5 and Proteasome signaling pathways) were overexpressed in vMCF-7 $7^{\mathrm{DNp} 53} 1 \mathrm{GX}$ cells compared to the vMCF-7 ${ }^{\mathrm{DNp} 53}$ parental cells. 

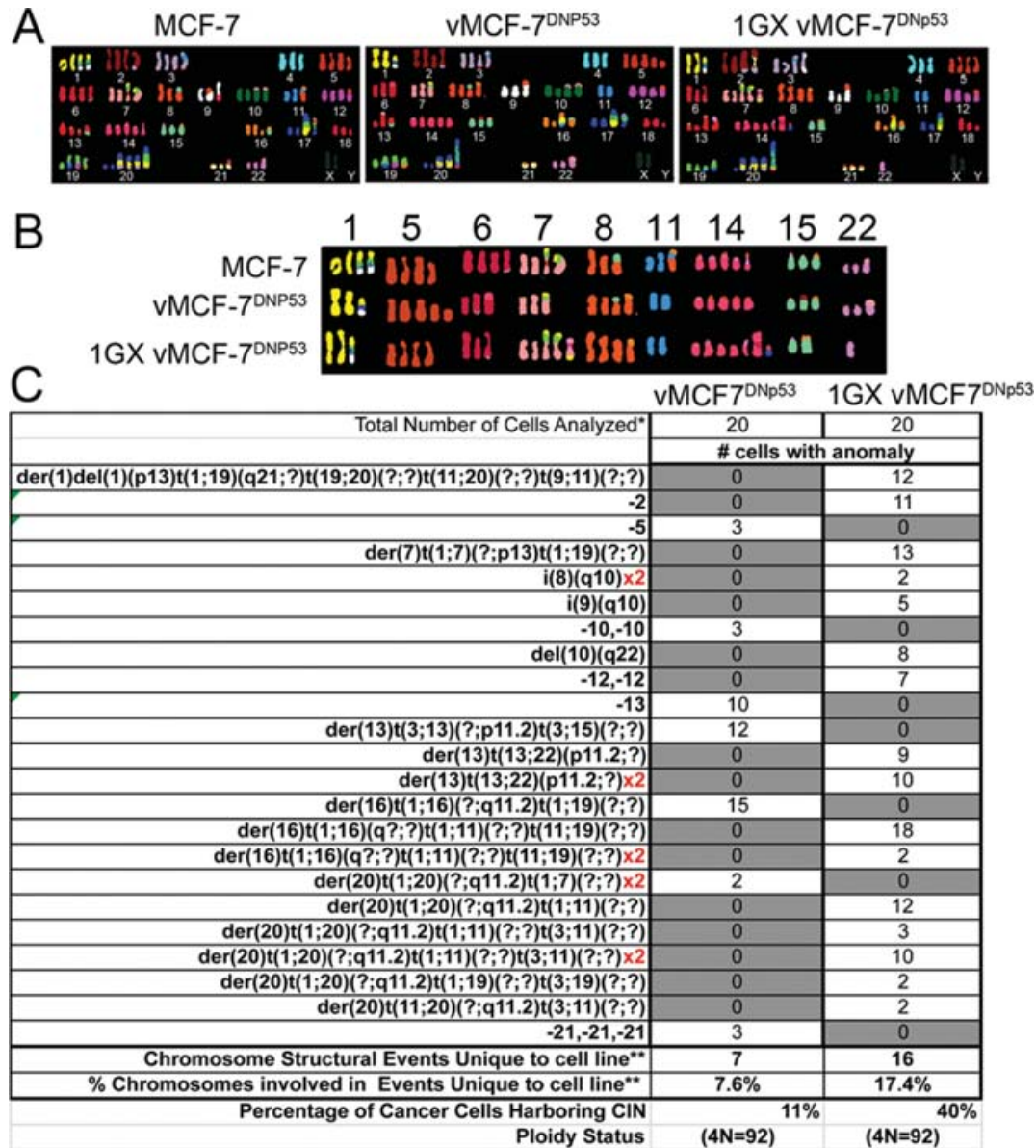

Figure 6. SKY and cytogenetic analysis of human breast cancer cell lines. Hybridization and detection of the human SKYPaint ${ }^{\circledR}$ probe were performed as recommended by the manufacturer. Image acquisition and spectral analysis of metaphase cells were achieved by using the SD200 SpectraCube ${ }^{\mathrm{TM}}$ Spectral Imaging system mounted on a Zeiss Axioplan2 microscope. Images were analyzed using HiSKY analysis software. (A) SKY analysis of MCF-7, vMCF$7^{\mathrm{DNp} 53}$ and vMCF-7 ${ }^{\mathrm{DNp} 53} 1 \mathrm{GX}$ cells. (B) Major gross chromosome abnormalities identified by SKY analysis of MCF-7, vMCF-7 ${ }^{\mathrm{DNp} 53}$ and vMCF-7 $7^{\mathrm{DN} 53} 1 \mathrm{GX}$ cells. (C) Comprehensive SKY and cytogenetic analysis showing structural and numerical chromosomal alterations unique to vMCF-7 ${ }^{\text {DNP53 }}$ and vMCF-7 ${ }^{\text {DNP53 }}$ $1 \mathrm{GX}$ cells compared to the parental MCF-7 cell line.

xenografts (vMCF-7 $\left.{ }^{\text {DNP53 }} 1 \mathrm{GX}\right)$. We have previously demonstrated that vMCF-7DNP53 xenografts develop high-grade breast tumors characterized by phenotypic heterogeneity for the estrogen receptor $\alpha(E R \alpha)$ and spontaneous metastatic spreading to the lung (12). Affimetrix microarray expression analysis revealed that 392 genes were uniquely expressed in vMCF-7 ${ }^{\text {DNP53 }} 1 \mathrm{GX}$ as compared to cultured vMCF-7 ${ }^{\mathrm{DNP53}}$ cells, suggesting that in vivo tumor growth leads to additional transcriptome changes (Fig. 4A and B).

Ingenuity pathways analysis of the 392 genes that arose during in vivo tumor growth revealed four additional noncanonical invasive-related networks (Fig. 4C and D and Fig. 5A and B) containing a total of 78 cancer genes in which nodal genes identified were involved in tumor self-renewal (SOX), chemoresistance and metastases (IGFB5, Proteasome, and p38MAPK signaling pathways). Importantly, these networks included the overexpression of AKT (EMT and chemoresistance), Cdk6 (cell proliferation), MUC1 (EMT and invasion), and down-regulation of Thbs 1 (inhibitor of angiogenesis), and Cdh18 (cadherin-18 adhesion molecule) genes, known to facilitate tumor progression and metastasis (26). These results demonstrate that breast cancer cells lacking an integral p53-signaling pathway are susceptible to develop invasive additional transcriptome signatures during tumor progression in vivo.

To establish whether the transcriptome changes observed exclusively in the vMCF-7 ${ }^{\mathrm{DNP53}} 1 \mathrm{GX}$ cells were linked to the development of chromosomal instability that arises during in vivo tumor growth, we performed an integrative karyotypic analysis of MCF-7, vMCF-7 ${ }^{\text {DNP53 }}$ and vMCF-7DNP53 1GX cells employing spectral karyotyping (SKY) technology and routine cytogenetic analysis (Fig. 4). Breast cancer cell lines were harvested and metaphase spreads for cytogenetic and SKY analyses were prepared as previously described $(23,27)$. Comparison of the three cell lines showed that each population had near-tetraploid karyotypes, yet harboring numerical and structural chromosomal aberrations that where distinct from one another (Fig. 6A). In particular, we identified gross chromosome gains and losses characteristic for MCF-7, vMCF-7 $7^{\text {DNp53 }}$ and vMCF-7 ${ }^{\text {DNp53 }} 1 \mathrm{GX}$ cells (Fig. 6B). These observations suggest that abrogation of p53 function results in chromosome alterations in vitro, which become further exacerbated during in vivo tumor growth. In order to identify unique structural and numerical chromosomal alterations 
Table I. Unique chromosomal anomalies linked to transcriptome changes in MCF-7 ${ }^{\mathrm{DNp} 53}$ cells.

\begin{tabular}{lllr}
\hline CHR. & \multicolumn{1}{c}{ Transcriptome } & \multicolumn{1}{c}{ Chromosomal anomaly } & No. of cells $^{\mathrm{a}}$ \\
\hline 1 & TRIM33, TGFB2, TSNAX & $\operatorname{der}(16) \mathrm{t}(1 ; 16)(? ; \mathrm{q} 11.2) \mathrm{t}(1 ; 19)(? ; ?)$ & 15 \\
& & $\operatorname{der}(20) \mathrm{t}(1 ; 20)(? ; \mathrm{q} 11.2) \mathrm{t}(1 ; 7)(? ; ?) \mathrm{x} 2$ & 2 \\
3 & LRIG1, KBTBD8, CPNE4, WWTR1 & $\operatorname{der}(13) \mathrm{t}(3 ; 13)(? ; \mathrm{p} 11.2) \mathrm{t}(3 ; 15)(? ; ?)$ & 12 \\
5 & NPR3, PPAP2A, FST, HBEGF & -5 & 3 \\
7 & EGFR, TBRG4, CALCR & $\operatorname{der}(20) \mathrm{t}(1 ; 20)(? ; \mathrm{q} 11.2) \mathrm{t}(1 ; 7)(? ; ?) \mathrm{x} 2$ & 2 \\
10 & PLXDC2, BAMBI, PLAU & $-10,-10$ & 3 \\
13 & EFNB2 & -13 & 10 \\
& & $\operatorname{der}(13) \mathrm{t}(3 ; 13)(? ; \mathrm{p} 11.2) \mathrm{t}(3 ; 15)(? ; ?)$ & 12 \\
15 & SMAD3 & $\operatorname{der}(13) \mathrm{t}(3 ; 13)(? ; \mathrm{p} 11.2) \mathrm{t}(3 ; 15)(? ; ?)$ & 12 \\
16 & CDH11 & $\operatorname{der}(16) \mathrm{t}(1 ; 16)(? ; \mathrm{q} 11.2) \mathrm{t}(1 ; 19)(? ; ?)$ & 15 \\
19 & (NONE) & $\operatorname{der}(16) \mathrm{t}(1 ; 16)(? ; \mathrm{q} 11.2) \mathrm{t}(1 ; 19)(? ; ?)$ & 15 \\
20 & JAG1, TGM2, BMP7 & $\operatorname{der}(20) \mathrm{t}(1 ; 20)(? ; \mathrm{q} 11.2) \mathrm{t}(1 ; 7)(? ; ?) \mathrm{x} 2$ & 2 \\
21 & (NONE) & $-21,-21,-21$ & 3 \\
\hline
\end{tabular}

a20 cells analyzed.

which were present in vMCF-7 ${ }^{\text {DNP53 }}$ and vMCF-7 ${ }^{\text {DNP53 }} 1 \mathrm{GX}$ cells in comparison to MCF-7 cells, we carried out a SKY and routine cytogenetic analysis (Fig. 6C), which revealed that vMCF-7 $7^{\mathrm{DNP} 53} 1 \mathrm{GX}$ cells display a higher percentage of chromosomal abnormalities $(17.4 \%)$ compared to vMCF-7DNP53 cells $(7.6 \%)$. To determine the rate of numerical and structural chromosomal alterations that characterize CIN, we analyzed the percentage of cells sharing identical chromosome abnormalities. A cell population with the same chromosome alteration pattern was considered clonal if it was representative of at least $10 \%$ of the total cells (1). vMCF-7 DNp53 $1 \mathrm{GX}$ displayed high karyotypic instability, with $40 \%$ of the cells showing non-clonal chromosome alteration patterns. In contrast, vMCF- $7^{\mathrm{DN} 533}$ cells showed relatively low karyotypic instability where only $11 \%$ of cells showed non-clonal chromosome alterations (Fig. 6C). These results indicate that in vivo tumor growth of vMCF-7 ${ }^{\text {DNP53 }}$ cells results in an increased level of chromosomal instability compared to the cultured vMCF-7DNP53 and to parental cells. These findings are consistent with our earlier observations of increased centrosome amplification, aberrant mitotic spindles, and phenotypic heterogeneity in vMCF-7DNP53 $1 \mathrm{GX}$ cells (12). Taken together, they also demonstrate a mechanistic linkage between centrosome amplification, development of CIN and consequent tumor cell heterogeneity.

Finally, we assessed the chromosomal location of the genes present in the non-canonical transcriptome networks and determined their relationship to unique chromosome abnormalities identified in the vMCF-7 ${ }^{\mathrm{DNp} 53}$ and vMCF- $7^{\mathrm{DNp} 53}$ $1 \mathrm{GX}$ cells (Fig. 6C). Importantly, this analysis revealed that $40 \%$ of genes identified in the vMCF-7 ${ }^{\mathrm{DNp} 53}$ transcriptome networks were linked to unique qualitative and quantitative chromosomal alterations, while this correlation increased to
$60 \%$ in vMCF-7 ${ }^{\text {DNp53 }} 1 \mathrm{GX}$ cells (Table I and II). These results demonstrate that abrogation of p53 function may drive the early transcriptome changes responsible for cell proliferation, EMT and survival, while further transcriptome changes that occur during in vivo tumor progression are mechanistically linked to the development of CIN providing the 'engine' for more invasive and metastatic properties.

Taken together, these observations demonstrate that impaired p53 function drives breast cancer progression through a multi-step process, characterized by the early development of EMT and during tumor progression by CIN responsible for the development of additional invasive transcriptome signatures. Specifically, we propose that during tumor progression increased chromosomal abnormalities in cancer cells lacking p53 function generate karyotypic variability leading to unique transcriptome signatures that are ultimately responsible for tumor progression and distant metastases. We identified novel non-canonical transcriptome networks involved in cell proliferation, EMT, chemoresistance and invasion that arise following abrogation of p53 function and development of CIN in breast cancer cells. Importantly, one of the non-canonical transcriptome networks was linked to the overexpression of the proteasome pathway responsible for the degradation of estrogen receptor (ER) and hormone independence in breast cancer cells (28). This result provides a possible explanation for the mechanistic role of centrosome amplification and $\mathrm{CI}$ in the loss of ER expression and consequent tumor cell heterogeneity in vMCF-7DNp53 $1 \mathrm{GX}$ cells that we have previously demonstrated (12). Finally, the identification of these cancer related transcriptome networks have important translational implications since some of the nodal genes identified in this study are 'druggable' making them suitable targets for biological therapies. For this reason 
Table II. Unique chromosomal anomalies linked to transcriptome changes in MCF-7 ${ }^{\text {DNp53 }} 1 \mathrm{GX}$ cells.

\begin{tabular}{|c|c|c|c|}
\hline CHR. & Transcriptome & Chromosomal anomaly & No. of cells ${ }^{\mathrm{a}}$ \\
\hline 1 & $\begin{array}{l}\text { SARS, ID3 } \\
\text { HSPG2, DNAJC6 } \\
\text { S100A9, SOX13 } \\
\text { CD55, TRAF5 } \\
\text { MUC1 } \\
\text { ELF3 } \\
\text { TGFB2 }\end{array}$ & $\begin{array}{l}\operatorname{der}(1) \operatorname{del}(1)(\mathrm{p} 13) \mathrm{t}(1 ; 19)(\mathrm{q} 21 ; ?) \mathrm{t}(19 ; 20)(? ; ?) \mathrm{t}(11 ; 20)(? ; ?) \mathrm{t}(9 ; 11)(? ; ?) \\
\operatorname{der}(7) \mathrm{t}(1 ; 7)(? ; \mathrm{p} 13) \mathrm{t}(1 ; 19)(? ; ?) \\
\operatorname{der}(16) \mathrm{t}(1 ; 16)(\mathrm{q} ? ; ?) \mathrm{t}(1 ; 11)(? ; ?) \mathrm{t}(11 ; 19)(? ; ?) \\
\operatorname{der}(16) \mathrm{t}(1 ; 16)(\mathrm{q} ? ; ?) \mathrm{t}(1 ; 11)(? ;) \mathrm{t}(11 ; 19)(? ; ?) \mathrm{x} 2 \\
\operatorname{der}(20) \mathrm{t}(1 ; 20)(? ; \mathrm{q} 11.2) \mathrm{t}(1 ; 11)(? ; ?) \\
\operatorname{der}(20) \mathrm{t}(1 ; 20)(? ; \mathrm{q} 11.2) \mathrm{t}(1 ; 11)(? ; ?) \mathrm{t}(3 ; 11)(? ; ?) \\
\operatorname{der}(20) \mathrm{t}(1 ; 20)(? ; \mathrm{q} 11.2) \mathrm{t}(1 ; 11)(? ; ?) \mathrm{t}(3 ; 11)(? ; ?) \times 2 \\
\operatorname{der}(20) \mathrm{t}(1 ; 20)(? ; \mathrm{q} 11.2) \mathrm{t}(1 ; 19)(? ; ?) \mathrm{t}(3 ; 19)(? ; ?)\end{array}$ & $\begin{array}{r}12 \\
13 \\
18 \\
2 \\
12 \\
3 \\
10 \\
2\end{array}$ \\
\hline 2 & $\begin{array}{l}\text { ACTG2, SERTAD2 } \\
\text { EIF2AK2, BCL2L11 } \\
\text { TFPI, DLX2 } \\
\text { DNAJC10, IGFBP5 } \\
\text { UBE2F }\end{array}$ & -2 & 11 \\
\hline 3 & SOX2 & $\begin{array}{l}\operatorname{der}(20) \mathrm{t}(1 ; 20)(? ; \mathrm{q} 11.2) \mathrm{t}(1 ; 11)(? ; ?) \mathrm{t}(3 ; 11)(? ; ?) \\
\operatorname{der}(20) \mathrm{t}(1 ; 20)(? ; \mathrm{q} 11.2) \mathrm{t}(1 ; 11)(? ; ?) \mathrm{t}(3 ; 11)(? ; ?) \mathrm{x} 2 \\
\operatorname{der}(20) \mathrm{t}(1 ; 20)(? ; \mathrm{q} 11.2) \mathrm{t}(1 ; 19)(? ; ?) \mathrm{t}(3 ; 19)(? ; ?) \\
\operatorname{der}(20) \mathrm{t}(11 ; 20)(? ; \mathrm{q} 11.2) \mathrm{t}(3 ; 11)(? ; ?)\end{array}$ & $\begin{array}{r}3 \\
10 \\
2 \\
2\end{array}$ \\
\hline 7 & CDK6, NUB1 & $\operatorname{der}(7) \mathrm{t}(1 ; 7)(? ; \mathrm{p} 13) \mathrm{t}(1 ; 19)(? ; ?)$ & 13 \\
\hline 8 & TACC1, MMP16 & $\mathrm{i}(8)(\mathrm{q} 10) \times 2$ & 2 \\
\hline 9 & HSPA5 & $\begin{array}{l}\mathrm{i}(9)(\mathrm{q} 10) \\
\operatorname{der}(1) \operatorname{del}(1)(\mathrm{p} 13) \mathrm{t}(1 ; 19)(\mathrm{q} 21 ; ?) \mathrm{t}(19 ; 20)(? ; ?) \mathrm{t}(11 ; 20)(? ; ?) \mathrm{t}(9 ; 11)(? ; ?)\end{array}$ & $\begin{array}{r}5 \\
12\end{array}$ \\
\hline 10 & MCM10, IKK & $\operatorname{del}(10)(q 22)$ & 8 \\
\hline 11 & $\begin{array}{l}\text { HSP70 } \\
\text { UBE2L6 } \\
\text { TSKU }\end{array}$ & $\begin{array}{l}\operatorname{der}(1) \operatorname{del}(1)(\mathrm{p} 13) \mathrm{t}(1 ; 19)(\mathrm{q} 21 ; ?) \mathrm{t}(19 ; 20)(? ; ?) \mathrm{t}(11 ; 20)(? ; ?) \mathrm{t}(9 ; 11)(? ; ?) \\
\operatorname{der}(16) \mathrm{t}(1 ; 16)(\mathrm{q} ? ; ?) \mathrm{t}(1 ; 11)(? ; ?) \mathrm{t}(11 ; 19)(? ; ?) \\
\operatorname{der}(16) \mathrm{t}(1 ; 16)(\mathrm{q} ? ; ?) \mathrm{t}(1 ; 11)(? ;) \mathrm{t}(11 ; 19)(? ; ?) \mathrm{x} 2 \\
\operatorname{der}(20) \mathrm{t}(1 ; 20)(? ; \mathrm{q} 11.2) \mathrm{t}(1 ; 11)(? ; ?) \mathrm{t}(3 ; 11)(? ; ?) \\
\operatorname{der}(20) \mathrm{t}(1 ; 20)(? ; \mathrm{q} 11.2) \mathrm{t}(1 ; 11)(? ; ?) \mathrm{t}(3 ; 11)(? ; ?) \times 2 \\
\operatorname{der}(20) \mathrm{t}(11 ; 20)(? ; \mathrm{q} 11.2) \mathrm{t}(3 ; 11)(? ; ?)\end{array}$ & $\begin{array}{r}12 \\
18 \\
2 \\
3 \\
10 \\
2\end{array}$ \\
\hline 12 & $\begin{array}{l}\text { C12ORF11, KITLG } \\
\text { HSP90B1 }\end{array}$ & $-12,-12$ & 7 \\
\hline 13 & $\begin{array}{l}\text { BRCA2, DLEU2 } \\
\text { KLF5, DNAJC3 }\end{array}$ & $\begin{array}{l}\operatorname{der}(13) t(13 ; 22)(\mathrm{p} 11.2 ; ?) \\
\operatorname{der}(13) \mathrm{t}(13 ; 22)(\mathrm{p} 11.2 ; ?) \times 2\end{array}$ & $\begin{array}{r}9 \\
10\end{array}$ \\
\hline 16 & FANCA & $\begin{array}{l}\operatorname{der}(16) \mathrm{t}(1 ; 16)(\mathrm{q} ? ; ?) \mathrm{t}(1 ; 11)(? ; ?) \mathrm{t}(11 ; 19)(? ; ?) \\
\operatorname{der}(16) \mathrm{t}(1 ; 16)(\mathrm{q} ? ; ?) \mathrm{t}(1 ; 11)(? ; ?) \mathrm{t}(11 ; 19)(? ; ?) \times 2\end{array}$ & $\begin{array}{r}18 \\
2\end{array}$ \\
\hline 19 & $\begin{array}{l}\text { GDF15 } \\
\text { HSH2D } \\
\text { TCF3 }\end{array}$ & $\begin{array}{l}\operatorname{der}(1) \operatorname{del}(1)(\mathrm{p} 13) \mathrm{t}(1 ; 19)(\mathrm{q} 21 ; ?) \mathrm{t}(19 ; 20)(? ; ?) \mathrm{t}(11 ; 20)(? ; ?) \mathrm{t}(9 ; 11)(? ; ?) \\
\operatorname{der}(7) \mathrm{t}(1 ; 7)(? ; \mathrm{p} 13) \mathrm{t}(1 ; 19)(? ; ?) \\
\operatorname{der}(16) \mathrm{t}(1 ; 16)(\mathrm{q} ? ;) \mathrm{t}(1 ; 11)(? ; ?) \mathrm{t}(11 ; 19)(? ; ?) \\
\operatorname{der}(16) \mathrm{t}(1 ; 16)(\mathrm{q} ? ; ?) \mathrm{t}(1 ; 11)(? ; ?) \mathrm{t}(11 ; 19)(? ; ?) \times 2\end{array}$ & $\begin{array}{r}12 \\
13 \\
18 \\
2\end{array}$ \\
\hline 20 & SNX5, THBDs & $\operatorname{der}(1) \operatorname{del}(1)(\mathrm{p} 13) \mathrm{t}(1 ; 19)(\mathrm{q} 21 ; ?) \mathrm{t}(19 ; 20)(? ; ?) \mathrm{t}(11 ; 20)(? ; ?) \mathrm{t}(9 ; 11)(? ; ?)$ & 12 \\
\hline 22 & (NONE) & $\begin{array}{l}\operatorname{der}(13) t(13 ; 22)(\mathrm{p} 11.2 ; ?) \\
\operatorname{der}(13) \mathrm{t}(13 ; 22)(\mathrm{p} 11.2 ; ?) \times 2\end{array}$ & $\begin{array}{r}9 \\
10\end{array}$ \\
\hline
\end{tabular}

a20 cells analyzed.

molecular targeting of EGF, TGF- 3 and the Proteasome signaling pathways represent a valid therapeutic rationale for tailoring the treatment of breast tumors that display mutant p53, EMT, CIN, and chemoresistance to ultimately improve the overall survival of breast cancer patients.

\section{Acknowledgements}

This study was supported by NCI CA72836 to J.L.S., USAMRMC BC022276 and Intramural RECDA Award to $\mathrm{ABD}$, the Italian Association for Cancer Research (AIRC) to 
A.A., the Mayo Clinic Breast Cancer Specialized Program of Research Excellence NIH CA116201 to J.I., and the Mayo Clinic School of Medicine. We also wish to acknowledge the Cytogenetics Shared Resource, a core facility of the Mayo Clinic Comprehensive Cancer Center, for performing SKY and routine cytogenetic analysis and assisting us with the interpretation of the results.

\section{References}

1. Lingle WL, Barrett SL, Negron VC, et al: Centrosome amplification drives chromosomal instability in breast tumor development. Proc Natl Acad Sci USA 99: 1978-1983, 2002.

2. D'Assoro AB, Lingle WL and Salisbury JL: Centrosome amplification and the development of cancer. Oncogene 21: 6146-6153, 2002.

3. Ashktorab H, Schaffer AA, Daremipouran M, Smoot DT, Lee E and Brim H: Distinct genetic alterations in colorectal cancer. PLoS ONE 5: E8879, 2010.

4. D'Assoro AB, Barrett SL, Folk C, et al: Amplified centrosomes in breast cancer: a potential indicator of tumor aggressiveness. Breast Cancer Res Treat 75: 25-34, 2002.

5. Fukasawa K: Oncogenes and tumour suppressors take on centrosomes. Nat Rev Cancer 7: 911-924, 2007.

6. Sluder G: Centrosome duplication and its regulation in higher animal cells. In: Centrosomes in Development and Disease. Nigg EA (ed). Wiley-VCH, Weinheim, pp167-190, 2004.

7. D'Assoro AB, Busby R, Suino K, et al: Genotoxic stress leads to centrosome amplification in breast cancer cell lines that have an inactive G1/S cell cycle checkpoint. Oncogene 23: 4068-4075, 2004.

8. Lingle WL, Lukasiewicz K and Salisbury JL: Deregulation of the centrosome cycle and the origin of chromosomal instability in cancer. Adv Exp Med Biol 570: 393-421, 2005.

9. Chuthapisith S, Eremin J, El-Sheemey M and Eremin O: Breast cancer chemoresistance: emerging importance of cancer stem cells. Surg Oncol 19: 27-32, 2009.

10. Lee HH, Zhu Y, Govindasamy KM and Gopalan G: Downregulation of Aurora-A overrides estrogen-mediated growth and chemoresistance in breast cancer cells. Endocr Relat Cancer 15: 765-775, 2008.

11. Chuthapisith S, Layfield R, Kerr ID, Hughes C and Eremin O: Proteomic profiling of MCF-7 breast cancer cells with chemoresistance to different types of anti-cancer drugs. Int J Oncol 30: 1545-1551, 2007.

12. D'Assoro AB, Busby R, Acu ID, et al: Impaired p53 function leads to centrosome amplification, acquired ERalpha phenotypic heterogeneity and distant metastases in breast cancer MCF-7 xenografts. Oncogene 27: 3901-3911, 2008.
13. Duensing A, Liu Y, Spardy N, et al: RNA polymerase II transcription is required for human papillomavirus type 16 E7and hydroxyurea-induced centriole overduplication. Oncogene 26: 215-223, 2007.

14. Menendez D, Inga A and Resnick MA: Estrogen receptor acting in cis enhances WT and mutant p53 transactivation at canonical and noncanonical p53 target sequences. Proc Natl Acad Sci USA 107: 1500-1505, 2010.

15. Hanashiro K, Kanai M, Geng Y, Sicinski P and Fukasawa K: Roles of cyclins $\mathrm{A}$ and $\mathrm{E}$ in induction of centrosome amplification in p53-compromised cells. Oncogene 27: 5288-5302, 2008.

16. Adon AM, Zeng X, Harrison MK, et al: Cdk2 and Cdk4 regulate the centrosome cycle and are critical mediators of centrosome amplification in p53-null cells. Mol Cell Biol 30: 694-710, 2009.

17. Scata KA and El-Deiry WS: p53, BRCA1 and breast cancer chemoresistance. Adv Exp Med Biol 608: 70-86, 2007.

18. Rakha EA, Elsheikh SE, Aleskandarany MA, et al: Triplenegative breast cancer: distinguishing between basal and nonbasal subtypes. Clin Cancer Res 15: 2302-2310, 2009.

19. Howard EM, Lau SK, Lyles RH, et al: Correlation and expression of p53, HER-2, vascular endothelial growth factor (VEGF), and e-cadherin in a high-risk breast-cancer population. Int $\mathrm{J}$ Clin Oncol 9: 154-160, 2004.

20. Medina D, Kittrell FS, Shepard A, Contreras A, Rosen JM and Lydon J: Hormone dependence in premalignant mammary progression. Cancer Res 63: 1067-1072, 2003.

21. Godar S, Ince TA, Bell GW, et al: Growth-inhibitory and tumor-suppressive functions of p53 depend on its repression of CD44 expression. Cell 134: 62-73, 2008.

22. Knippschild U, Oren M and Deppert W: Abrogation of wildtype p53 mediated growth-inhibition by nuclear exclusion. Oncogene 12: 1755-1765, 1996.

23. Spurbeck JL, Carlson RO, Allen JE and Dewald GW: Culturing and robotic harvesting of bone marrow, lymph nodes, peripheral blood, fibroblasts, and solid tumors with in situ techniques. Cancer Genet Cytogenet 32: 59-66, 1988.

24. Schrock E, du Manoir S, Veldman T, et al: Multicolor spectral karyotyping of human chromosomes. Science 273: 494-497, 1996.

25. Barch M, Knutsen T and Spurbeck J: The AGT Cytogenetics Laboratory Manual III. Lippincott-Raven Press, Philadelphia, 1997.

26. Polyak K and Weinberg RA: Transitions between epithelial and mesenchymal states: acquisition of malignant and stem cell traits. Nat Rev Cancer 9: 265-273, 2009.

27. Spurbeck JL, Zinsmeister AR, Meyer KJ and Jalal SM: Dynamics of chromosome spreading. Am J Med Genet 61: 387-393, 1996.

28. Messaoudi S, Peyrat JF, Brion JD and Alami M: Recent advances in Hsp90 inhibitors as antitumor agents. Anticancer Agents Med Chem 8: 761-782, 2008 